\title{
Correction to: The modernity of Dedekind's anticipations contained in What are numbers and what are they good for?
}

\section{J. Climent Vidal ${ }^{1}$ (D) J. Soliveres Tur ${ }^{1}$}

Abstract The original version of this article unfortunately contained a mistake: The equation on page 34 was incorrect. The corrected equation is given below.

\section{Correction to: Arch. Hist. Exact Sci. https://doi.org/10.1007/s00407-018-0202-6}

The original version of this article unfortunately contained a mistake: The equation on page 34 was incorrect. The corrected equation is given below.

$$
\Sigma_{w, n}^{\mathrm{pr}}= \begin{cases}\left\{\kappa_{0,0}\right\}, & \text { if } w=\lambda \text { and } n=0 ; \\ \{\mathrm{sc}\} \cup\left\{\mathrm{pr}_{1,0}\right\}, & \text { if } w=\lambda \text { and } n=1 ; \\ \left\{\mathrm{pr}_{n, i} \mid i \in n\right\}, & \text { if } w=\lambda \text { and } n \geq 2 ; \\ \left\{\Omega_{\mathrm{C}}^{m, n}\right\}, & \text { if } w=(m) \curlywedge(n \mid i \in m) \text { and } m \geq 1 ; \\ \left\{\Omega_{\mathrm{R}}^{m}\right\}, & \text { if } w=(m) \curlywedge(m+2) \text { and } n=m+1 ; \\ \varnothing, & \text { otherwise. }\end{cases}
$$

The original article has been corrected.

The original article can be found online at https://doi.org/10.1007/s00407-018-0202-6.

\footnotetext{
$凶 \quad$ J. Climent Vidal

Juan.B.Climent@uv.es

J. Soliveres Tur

Juan.Soliveres@uv.es
}

1 Departamento de Lógica y Filosofía de la Ciencia, Universidad de Valencia, Avda. Blasco Ibáñez, 30-7a, 46010 Valencia, Spain 